\title{
El efecto del liderazgo, aprendizaje organizacional y administración del conocimiento en la percepción de la innovación del personal operativo en la Ciudad de México
}

\author{
The effect of leadership, organizational learning, and knowledge management \\ on the perception of innovation by operational personnel in Mexico City
}

\author{
Víctor Manuel Roque López* y Antonio Alejandro Arriaga Martínez \\ Universidad Anáhuac, México
}

Recibido el 16 de junio de 2017; aceptado el 1 de enero de 2018

Disponible en Internet el: 6 de diciembre de 2018

\section{Resumen}

El objetivo de este estudio es ofrecer una explicación de los factores que tienen un impacto en la percepción de la innovación del personal operativo en empresas mexicanas. Mediante un estudio exploratorio, se detectaron 26 atributos de la innovación. Se diseñó un instrumento para medir la percepción sobre el cumplimiento de estos atributos. Se integró una muestra de 925 personas de nivel operativo de empresas mexicanas manufactureras y de servicio. Los atributos se agruparon en cuatro dimensiones mediante un análisis factorial confirmatorio. Para analizar el impacto de las dimensiones en la innovación se desarrollaron: un modelo de regresión y dos modelos estructurales. Los análisis estructurales arrojaron que los factores aprendizaje organizacional, administración del conocimiento y liderazgo, generan un impacto significativo en la percepción de la innovación por parte del personal operativo. De estos factores, el liderazgo obtuvo los pesos estadísticos más altos en los modelos estructurales y un efecto significativo sobre la innovación.

Código JEL: M10, M13, M16

Palabras clave: Innovación; Desempeño organizacional; Empresas mexicanas

\footnotetext{
* Autor para correspondencia

Correo electrónico: vroquel@hotmail.com (V.M. Roque López)

La revisión por pares es responsabilidad de la Universidad Nacional Autónoma de México
} 


\begin{abstract}
The objective of this study is to provide an explanation of the factors that have an impact on the perception of innovation in Mexican companies. Through an exploratory study, 26 attributes of innovation were detected. An instrument was designed in order to measure the perception of compliance with these attributes. A sample of $n=925$ people of operational level of Mexican manufacturing and service companies was conformed. To assess the impact of the factors on innovation, a regression model and two structural models were developed. The analysis of structural models showed that the factors organizational learning, knowledge management and leadership tend to have a significant impact on the perception of innovation by the operational staff. From these factors, leadership achieved the highest statistical weights in structural models and a significant effect on innovation.
\end{abstract}

JEL codes: M10, M13, M16

Keywords: Innovation; Organizational performance; Mexican companies

\title{
Introducción
}

El estudio de la innovación ha mostrado una evolución en el tiempo en su conceptualización, incorporando elementos para precisar sus características y abordando varios niveles de análisis como se puede ver en: Schumpeter (1934, 1942), Jiménez-Jiménez y Sanz-Valle (2011), García-Morales et al. (2008, 2012), Grigoriou y Rothaermel (2014), Christensen (1997), y en Christensen et al. (2015), sólo por mencionar a algunos.

Los factores que tienden a tener un efecto importante en la innovación son variados y han sido abordados por diversos autores en estudios previos, siendo relevantes para el presente estudio el liderazgo (García-Morales et al., 2008, 2012; Noruzy et al., 2013; entre otros autores), el aprendizaje organizacional (Jiménez-Jiménez y Sanz-Valle, 2011; Hu, 2014; entre otros autores), y la administración del conocimiento (Wu et al., 2014; Ugalde-Binda et al., 2014; entre otros autores).

De esta forma, el propósito de este trabajo apunta a analizar si existe incidencia de los factores liderazgo, aprendizaje organizacional y administración del conocimiento en la innovación del personal operativo en las empresas mexicanas. Con esto se abona a la comprensión de la innovación en el contexto del personal operativo en México, así como en la identificación de los principales factores involucrados para que se presente la innovación en las empresas mexicanas. El trabajo desarrollado es pionero en el contexto de México, dado que la gran mayoría de los estudios revisados han sido desarrollados en otros países y el desarrollo de esta investigación a diferencia de las demás, tomó en cuenta la perspectiva del personal operativo en las empresas mexicanas.

\section{Liderazgo}

El liderazgo considera las definiciones de García-Morales et al. $(2012,2008)$ quienes se enfocan en el liderazgo transformacional que es un estilo de liderazgo que realza la conciencia del interés colectivo entre los miembros de la organización y los ayuda a alcanzar sus metas colectivas. Por otra parte, Bass y Avolio (1999) se enfocan en el liderazgo transaccional el cual se enfoca en promover los intereses individuales de los líderes y sus seguidores para alcanzar 
la satisfacción de las obligaciones contractuales de parte de ambos, a través del establecimiento de objetivos y el monitoreo y control de los resultados. Adicionalmente, Graen et al. (1982) observan el liderazgo transaccional (Líder-Miembro-Intercambio) aplicando una perspectiva a la teoría del liderazgo fundamentada en diadas e intercambios y Scandura y Graen (1984) evalúan la calidad de la relación de intercambio líder-miembro, en particular entre el supervisor inmediato y el participante del estudio mediante cuestionarios con una escala validada en investigaciones previas de liderazgo e innovación.

\section{Aprendizaje organizacional}

El aprendizaje organizacional se fundamenta en la definición de Jiménez-Jiménez y SanzValle (2011), quienes consideran que el aprendizaje organizacional es un proceso por el cual la firma desarrolla nuevo conocimiento y entendimientos a partir de las experiencias de la gente en la organización, y señalan que el aprendizaje tiene un potencial para influir en los comportamientos y mejorar las capacidades de la firma. Bajo esta perspectiva, el aprendizaje organizacional es la base para adquirir una ventaja competitiva sustentable y una variable clave en el impulso del desempeño organizacional. Las firmas que son capaces de aprender tienen mejores posibilidades de identificar eventos y tendencias en el mercado y, en consecuencia, son firmas que son más flexibles y responden de forma más rápida a los nuevos desafíos que los competidores, lo que permite a las firmas mantener sus ventajas competitivas en el largo plazo.

\section{Administración del conocimiento}

La administración del conocimiento se basa en la aproximación de Wang y Lin (2013), quienes consideran que la administración del conocimiento es una propensión relativa de la organización a construir en su conocimiento alcanzado (memoria), así como a compartir, asimilar (absorber) y ser receptiva al nuevo conocimiento. Bajo esta perspectiva, la memoria organizacional es la información que viene de la historia de la organización y suele influir en las decisiones; compartir el conocimiento se refiere a la transferencia de conocimiento, habilidades y tecnología entre las subunidades de la organización; la absorción es la habilidad para reconocer el valor del nuevo conocimiento, asimilarlo y aplicarlo; y la receptividad refleja la facilidad con la que nuevas ideas son tomadas al interior de la firma.

\section{Innovación}

La innovación se aborda en el presente estudio considerando varias aproximaciones. Desde el punto de vista de los recursos con que cuenta la firma, Tsai y Yang (2014) consideran que la capacidad de innovación de la firma es la apertura permanente a las nuevas ideas como un aspecto de la cultura de la firma. García-Morales et al. $(2012,2008)$ utilizan la definición formulada por la Asociación de Desarrollo y Administración del Producto que analiza la innovación como una nueva idea, método o dispositivo, el acto de crear un nuevo producto, servicio o proceso. Cepeda-Carrion et al. (2012 señalan que la capacidad de innovación de la firma involucra el grado de apoyo y la permeabilidad hacia la innovación en la organización en términos del desarrollo de nuevos productos o procesos, la apertura de nuevos mercados o el desarrollo de una nueva dirección estratégica. Akman y Yilmaz (2008) definen la capacidad 
innovadora como un factor importante que facilita una cultura organizacional innovadora, considerando las características de las actividades promotoras internas y las capacidades de entendimiento y respuesta apropiadas hacia el medio ambiente externo.

\section{Medición de la innovación y sus factores}

Se consideraron las aportaciones de varios autores que se muestran en la Tabla 1.

Tabla 1

Relación de autores y aproximación teórica.

\begin{tabular}{|c|c|c|c|}
\hline Dimensión & Autor & Visualización & $\begin{array}{l}\text { Variable } \\
\text { dependiente }\end{array}$ \\
\hline Innovación & $\begin{array}{l}\text { Anderson et } \\
\text { al. (2014) } \\
\text { McKinley et } \\
\text { al. (2014) } \\
\text { Seidel y } \\
\text { O’Mahony } \\
\text { (2014) } \\
\text { Alexander } \\
\text { y Van } \\
\text { Knippenberg } \\
\text { (2014) } \\
\\
\text { Verre et al. } \\
\text { (2014) }\end{array}$ & $\begin{array}{l}\text { Definición integradora de innovación que considera a la } \\
\text { creatividad y a la innovación como partes esenciales del } \\
\text { mismo proceso y aplican un marco de estudio de niveles } \\
\text { de análisis para revisar la investigación en innovación. } \\
\text { Innovaciones flexibles e inflexibles como factores que } \\
\text { pueden llevar a un cambio organizacional y construyen } \\
\text { cuatro escenarios para las organizaciones que innovan o } \\
\text { responden de forma rígida o flexible. } \\
\text { Prácticas para producir unión en el concepto del } \\
\text { producto y lograr el entendimiento de los atributos } \\
\text { deseados para apoyar la coordinación de la innovación } \\
\text { y en especial las tareas de diseño del producto. } \\
\text { Impulsores de la innovación que permiten a los equipos } \\
\text { de trabajo enfrentar efectivamente los desafíos en el } \\
\text { desarrollo de innovaciones radicales como una alta } \\
\text { incertidumbre y el riesgo de fallar, así como desafíos no } \\
\text { anticipados que requieren la concertación de esfuerzos } \\
\text { de los equipos. } \\
\text { Estrategia de apropiabilidad del valor de la innovación } \\
\text { tecnológica y los riesgos asociados con el apoyo de } \\
\text { las fuentes externas de conocimiento. La cooperación } \\
\text { pública-privada para la innovación genera es importante } \\
\text { para la evaluación de los efectos de la estrategia de } \\
\text { apropiación. }\end{array}$ & $\begin{array}{l}\text { Innovación } \\
\text { (Definición) } \\
\text { Innovación } \\
\text { (Capacidades) } \\
\text { Innovación } \\
\text { (Capacidades) } \\
\text { Innovación } \\
\text { (Impulsores) } \\
\\
\text { Innovación } \\
\text { (Apropiación del } \\
\text { valor) }\end{array}$ \\
\hline
\end{tabular}




\begin{tabular}{|c|c|c|c|}
\hline Dimensión & Autor & Visualización & $\begin{array}{l}\text { Variable } \\
\text { Independiente }\end{array}$ \\
\hline $\begin{array}{l}\text { Aprendizaje } \\
\text { Organizacional }\end{array}$ & $\begin{array}{l}\text { Wang et al. } \\
\text { (2014) } \\
\text { Funk (2014) } \\
\text { Molina- } \\
\text { Morales et al. } \\
\text { (2014) } \\
\text { Hu (2014) } \\
\text { Cepeda- } \\
\text { Carrion et al. } \\
\text { (2012) } \\
\text { Jiménez- } \\
\text { Jiménez y } \\
\text { Sanz-Valle } \\
\text { (2011) } \\
\text { Yeung et al. } \\
\text { (2007) }\end{array}$ & $\begin{array}{l}\text { La innovación está doblemente incrustada en una red } \\
\text { social de colaboración entre investigadores y en una red } \\
\text { de conocimiento compuesta por ligas entre elementos } \\
\text { del conocimiento. Se presentan dos elementos para } \\
\text { la innovación: los vacíos estructurales y el grado de } \\
\text { centralidad en las redes. } \\
\text { La proximidad geográfica de los colegas en una } \\
\text { industria puede fomentar la generación de innovación } \\
\text { por parte de las empresas. Los efectos sin moderados } \\
\text { por la red de estructuras intraorganizacionales. } \\
\text { La proximidad cognitiva oen términos de metas y cultura } \\
\text { dirige a las firmas pertenecientes a un conglomerado } \\
\text { territorial a alcanzar la adquisición del conocimiento, } \\
\text { obteniendo como resultado una innovación relevante. } \\
\text { El aprendizaje organizacional como mediador total en } \\
\text { la relación entre los modelos de negocios centrados en } \\
\text { la eficiencia y la innovación tecnológica y mediador } \\
\text { parcial entre los modelos de negocios centrados en la } \\
\text { novedad y la innovación tecnológica. } \\
\text { Capacidad de absorción del conocimiento como un } \\
\text { factor determinante para desarrollar la innovación y } \\
\text { se identifican contextos potenciales y capacidades que } \\
\text { pueden actuar como sus impulsores. } \\
\text { El aprendizaje organizacional afecta de forma positiva al } \\
\text { desempeño y a la innovación. El efecto del aprendizaje } \\
\text { organizacional y el efecto de la innovación contribuyen } \\
\text { positivamente al desempeño del negocio. } \\
\text { Impacto del aprendizaje organizacional en la innovación, } \\
\text { la eficiencia interna, la satisfacción del consumidor, y } \\
\text { el desempeño financiero. Los impactos del aprendizaje } \\
\text { organizacional en las variables dependen de los } \\
\text { contextos organizacionales de la firma. }\end{array}$ & $\begin{array}{l}\begin{array}{l}\text { Aprendizaje } \\
\text { organizacional } \\
\text { (Redes) }\end{array} \\
\text { Aprendizaje } \\
\text { organizacional } \\
\text { (Proximidad } \\
\text { geográfica) } \\
\text { Aprendizaje } \\
\text { organizacional } \\
\text { (Proximidad } \\
\text { cognitiva } \\
\text { Aprendizaje } \\
\text { organizacional } \\
\text { (Modelos de } \\
\text { negocios) } \\
\text { Aprendizaje } \\
\text { organizacional } \\
\text { (Absorción) } \\
\text { Aprendizaje } \\
\text { organizacional } \\
\text { (Impulsor) } \\
\text { Aprendizaje } \\
\text { organizacional } \\
\text { (Contexto } \\
\text { organizacional) }\end{array}$ \\
\hline
\end{tabular}




\begin{tabular}{|c|c|c|c|}
\hline Dimensión & Autor & Visualización & $\begin{array}{l}\text { Variable } \\
\text { Independiente }\end{array}$ \\
\hline $\begin{array}{l}\text { Administración } \\
\text { del conocimiento }\end{array}$ & $\begin{array}{l}\text { Grigoriou y } \\
\text { Rothaermel } \\
\text { (2014) } \\
\text { Rogan and } \\
\text { Mors (2014) } \\
\text { Capaldo et al. } \\
\text { (2014) } \\
\text { Tuertscher et } \\
\text { al. (2014) } \\
\text { Wu et al. } \\
\text { (2014 } \\
\text { Ugalde-Binda } \\
\text { et al. (2014) }\end{array}$ & $\begin{array}{l}\text { Relaciones entre los individuos para desempeñarse } \\
\text { efectivamente en las actividades de generación de } \\
\text { conocimiento, apoyándose en las redes de conocimiento } \\
\text { intraorganizacionales que emergen a través de la } \\
\text { colaboración individual. } \\
\text { Las redes de contactos de los administradores para } \\
\text { obtener conocimiento e información como impulsores } \\
\text { de sus habilidades para balancear la decisión entre } \\
\text { explorar nuevos negocios y explotar los negocios } \\
\text { existentes. } \\
\text { Perspectiva contingente del valor de la innovación con } \\
\text { la distancia y madurez del conocimiento en la industria. } \\
\text { Las colaboraciones para desarrollar y desplegar } \\
\text { sistemas tecnológicos complejos y que implican una } \\
\text { experimentación y ajustes pueden servir como base para } \\
\text { la transformación organizacional. } \\
\text { La necesidad de cognición o la tendencia del individuo a } \\
\text { comprometerse y disfrutar el pensamiento, está asociada } \\
\text { con el comportamiento individual innovador. } \\
\text { La influencia del capital intelectual y las características } \\
\text { personales de los emprendedores en los resultados } \\
\text { de innovación presentan una relación positiva y } \\
\text { significativa. }\end{array}$ & $\begin{array}{l}\text { Administración } \\
\text { del conocimiento } \\
\text { (Redes de } \\
\text { conocimiento) } \\
\text { Administración } \\
\text { del conocimiento } \\
\text { (Conocimiento e } \\
\text { información) } \\
\text { Administración del } \\
\text { conocimiento (Valor } \\
\text { de la innovación) } \\
\text { Administración } \\
\text { del conocimiento } \\
\text { (Colaboración) } \\
\text { Administración } \\
\text { del conocimiento } \\
\text { (Cognición) } \\
\text { Administración } \\
\text { del conocimiento } \\
\text { (Capital intelectual) }\end{array}$ \\
\hline
\end{tabular}




\begin{tabular}{|c|c|c|c|}
\hline Dimensión & Autor & Visualización & $\begin{array}{l}\text { Variable } \\
\text { independiente }\end{array}$ \\
\hline Liderazgo & $\begin{array}{l}\text { Noruzy et al. } \\
(2013) \\
\text { Wallace et al. } \\
(2013)\end{array}$ & $\begin{array}{l}\text { Relaciones entre el liderazgo transformacional, } \\
\text { el aprendizaje organizacional, administración del } \\
\text { conocimiento, innovación organizacional y desempeño } \\
\text { organizacional entre empresas de manufacturas. } \\
\text { Efectos del clima de involucramiento del empleado } \\
\text { en el proceso de innovación que se desarrolla a nivel } \\
\text { individual, ligando el enfoque en la regulación del } \\
\text { empleado a la innovación a través del sentido de } \\
\text { vitalidad y aprendizaje en el trabajo. } \\
\text { Los esfuerzos de investigación y desarrollo no oficial } \\
\text { o informal ayudan a los individuos a desarrollar } \\
\text { innovaciones basadas en la exploración de territorio no } \\
\text { mapeado y en la evaluación demorada de las ideas en } \\
\text { etapa embrionaria. } \\
\text { Influencias del liderazgo transformacional en el } \\
\text { desempeño organizacional a través de las capacidades } \\
\text { dinámicas del aprendizaje organizacional y la } \\
\text { innovación. } \\
\text { Relación entre cultura e innovación, considerando que } \\
\text { existe un efecto directo e indirecto de la cultura en la } \\
\text { innovación a través del emprendimiento de nuevos } \\
\text { negocios. } \\
\text { Interrelaciones entre el liderazgo transformacional y } \\
\text { el desempeño organizacional a través de los efectos } \\
\text { de la generación y la revelación del conocimiento, } \\
\text { la capacidad de absorción del conocimiento, el } \\
\text { conocimiento tácito, el aprendizaje organizacional y la } \\
\text { innovación. }\end{array}$ & $\begin{array}{l}\text { Liderazgo (Impulsor } \\
\text { de la innovación) } \\
\text { Liderazgo (Clima } \\
\text { organizacional) } \\
\text { Liderazgo (Cultura) } \\
\text { Liderazgo (Impulsor } \\
\text { del desempeño) } \\
\text { Liderazgo (Cultura) } \\
\text { Liderazgo (Impulsor } \\
\text { del aprendizaje } \\
\text { organizacional y la } \\
\text { innovación) }\end{array}$ \\
\hline
\end{tabular}

Fuente: Desarrollado por los autores. 


\section{Efecto del liderazgo, aprendizaje organizacional y administración del conocimiento en la percepción de la innovación.}

\section{Efecto del liderazgo en la innovación}

Noruzy et al. (2013) determinan las relaciones entre el liderazgo transformacional, el aprendizaje organizacional, administración del conocimiento, innovación organizacional y desempeño organizacional. La relación entre cultura e innovación es estudiada mediante un estudio empírico por Nissan et al. (2012). Wallace et al. (2013) examinan los efectos del clima de involucramiento del empleado en el proceso de innovación que se desarrolla a nivel individual. Criscuolo et al. (2013) que los esfuerzos informales o no oficiales de los individuos están asociados con el alcance de altos niveles de desempeño innovador. Adicionalmente, se analizan las influencias del liderazgo transformacional en el desempeño organizacional a través de las capacidades dinámicas del aprendizaje organizacional y la innovación, corroborándose de forma empírica las influencias identificadas teóricamente (García-Morales et al., 2012). García-Morales et al. (2008) estudian las interrelaciones entre el liderazgo transformacional y el desempeño organizacional a través de los efectos de la generación y la revelación del conocimiento, la capacidad de absorción del conocimiento, el conocimiento tácito, el aprendizaje organizacional y la innovación.

\section{Efecto del aprendizaje organizacional en la innovación}

Wang et al. (2014) señalan que la innovación está doblemente incrustada en una red social de colaboración entre investigadores y en una red de conocimiento compuesta por ligas entre elementos del conocimiento. Se ha encontrado que, aunque la proximidad geográfica de los colegas en una industria puede fomentar el desempeño, los efectos son moderados por la red de estructuras intraorganizacionales (Funk ,2014). Asimismo, Molina-Morales et al. (2014) exploran la influencia relativa de la proximidad geográfica y cognitiva para explicar el desempeño en la innovación. Hu (2014) examina el efecto de los modelos de negocios en el desempeño en la innovación tecnológica a través de un rol mediador del aprendizaje organizacional. CepedaCarrion et al. (2012) consideran a la capacidad de absorción del conocimiento como un factor determinante para desarrollar la innovación e identifican contextos potenciales y capacidades que pueden actuar como sus impulsores. Jiménez-Jiménez y Sanz-Valle (2011) observan una relación positiva entre aprendizaje organizacional tanto con el desempeño como con la innovación. Yeung et al. (2007) estudian el impacto que presenta el aprendizaje organizacional en la innovación, la eficiencia interna, la satisfacción del consumidor, y el desempeño financiero.

\section{Efecto de la administración del conocimiento en la innovación}

Grigoriou y Rothaermel (2014) enfatizan la importancia de las relaciones entre los individuos para desempeñarse efectivamente en las actividades de generación de conocimiento. Rogan y Mors (2014) identifican a las redes de contactos de los administradores para obtener conocimiento e información como impulsores de sus habilidades para la toma de decisiones. Capaldo et al. (2014) señalan que el valor científico de una innovación se incrementa con la madurez en el conocimiento en el cual está basado, pero más allá de ese punto el valor declina. 
Tuertscher et al. (2014) estudian las colaboraciones entre múltiples partes involucradas para desarrollar y desplegar sistemas tecnológicos complejos. Wu et al. (2014) proponen que la necesidad de cognición o la tendencia del individuo a comprometerse y disfrutar el pensamiento, está asociada con el comportamiento individual innovador.

La tabla 2 muestra los autores de referencia para la medición de los factores del presente trabajo. La primera columna muestra los conceptos y la segunda reporta los autores y fecha de publicación.

Tabla 2

Conceptos y autores de referencia para la medición.

\begin{tabular}{ll}
\hline Concepto de estudio & Autores y fecha \\
\hline Innovación & $\begin{array}{l}\text { García-Morales et al. (2012), García-Morales et al. (2008), Cepeda-Carrion et } \\
\text { al. (2012), Akman y Yilmaz (2008), Tsai y Yang (2014). }\end{array}$ \\
\hdashline Aprendizaje organizacional & Jiménez-Jiménez y Sanz-Valle (2011). \\
\hline Administración del conocimiento & Wang y Lin (2013). \\
\hdashline Liderazgo & $\begin{array}{l}\text { García-Morales et al. (2012), García-Morales et al. (2008), Bass y Avolio } \\
\text { (1999), Graen et al. (1982), Scandura y Graen (1984). }\end{array}$ \\
\hline
\end{tabular}

Fuente: Desarrollado por los autores.

\section{Planteamiento}

De forma preliminar se llevó a cabo un estudio exploratorio con el propósito de detectar los factores que actúan sobre la empresa para la generación de la innovación. Como parte de este estudio preliminar se realizaron entrevistas a profundidad a 20 empleados y adicionalmente con la revisión de la literatura se detectaron los factores que actúan sobre la empresa para la generación de la innovación.

De esta forma se plantearon 3 hipótesis, en las cuales se espera que exista un efecto positivo de cada uno de los factores hacia la innovación.

Estas hipótesis se esquematizan en la Figura 1.

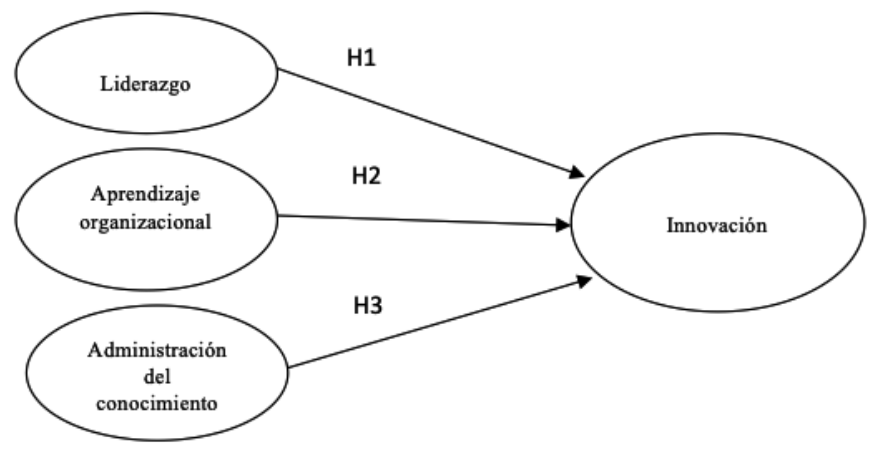

Figura 1. Planteamiento e hipótesis iniciales. Fuente: Desarrollado por los autores. 
Aunque existen más factores que pueden incidir en la innovación, en el caso del presente estudio la relación sólo se plantea entre el liderazgo, aprendizaje organizacional y administración del conocimiento (como variables independientes) y la innovación como variable dependiente final.

\section{Metodología}

Debido a que el objetivo del estudio es determinar la incidencia de los factores anteriormente señalados, se llevó a cabo un diseño de la investigación correlacional-causal de tipo no experimental (Kerlinger, 1979; Kerlinger y Lee, 2002; Hernández et al., 2010). Como se mencionó anteriormente, se realizaron entrevistas a profundidad a 20 empleados y adicionalmente con la revisión de la literatura se detectaron los factores que actúan para la generación de la innovación y se desarrolló un instrumento para la recolección de datos. Se detectaron veintiséis atributos en total.

Los reactivos utilizados para medir cada uno de los conceptos, como aparecen en el cuestionario pueden verse en la Tabla 3. Las preguntas se presentaron de forma aleatoria respecto de cada concepto en el estudio, y los reactivos fueron asociados a una escala de Likert de siete categorías de respuesta $(1=$ en total desacuerdo, $2=$ en desacuerdo, $3=$ ligeramente en desacuerdo, 4 = ni de acuerdo ni en desacuerdo, $5=$ ligeramente de acuerdo, $6=$ de acuerdo, 7 = en total acuerdo). El cuestionario se diseñó para ser aplicado al personal operativo y evaluar su percepción acerca de la innovación en la empresa. Se conformó una muestra de $\mathrm{n}=925$ personas de nivel operativo seleccionados por un procedimiento no probabilístico de muestreo por conveniencia y considerando diferentes zonas de la Ciudad de México en empresas de los rubros de manufactura y servicios.

\section{Análisis preliminar de datos}

Para el análisis de datos, primero se llevó a cabo el análisis factorial confirmatorio para corroborar una separación coherente de los conceptos incluyendo las mediciones de innovación. De esta forma se generaron cuatro componentes (dimensiones) aprendizaje organizacional, administración del conocimiento, liderazgo y una dimensión de innovación que posteriormente tuvo la función de variable dependiente latente en el análisis estructural. La integración de estos componentes producto del análisis factorial puede verse en la Tabla 3. Esta separación coherente de conceptos provee evidencia sobre la validez de medición de los constructos y de las dimensiones (variables latentes) resultantes. 
Tabla 3

Análisis factorial confirmatorio de las dimensiones del modelo. Rotación varimax. Cargas factoriales (matriz patrón)

Componentes

Atributos (variable observada)

1

.49

Se conocen las fortalezas y debilidades del personal de la organización.

La empresa comparte los cambios a realizar en su operación.

Se conocen los nuevos productos o servicios ofrecidos por nuestra empresa.

Todos los miembros de la organización comparten la misma meta hacia la cual se sienten comprometidos.

Los empleados comparten conocimientos y experiencias en su área de trabajo comunicándose entre ellos.

Evaluamos rápidamente las nuevas ideas que surgen en nuestra empresa.

Frecuentemente compartimos ideas con otras personas de intereses comunes aunque estén en diferentes áreas.

Se difunden nuevas ideas para mejorar la operación de la organización.

Se realizan reuniones o juntas donde las personas comparten conocimientos y aprenden unos de otros.

Se comparte el conocimiento y experiencias entre los empleados de diferentes áreas.

Al compartir la información y el conocimiento frecuentemente generamos nuevas ideas para mejorar nuestro negocio.

La empresa cuenta con información actualizada de sus clientes atendidos.

Mi supervisor dedica tiempo a enseñarme y/o asesorarme en mis actividades laborales.

Hay suficiente confianza con mi supervisor para aceptar y retroalimentar sus decisiones.

Confío en las recomendaciones de mi supervisor para mejorar como empleado.

La dirección de la empresa motiva al personal para alcanzar sus objetivos.

El gerente motiva a los empleados para mejorar las actividades desarrolladas en la organización.

La organización cuenta con líderes que son capaces de motivar y guiar a sus compañeros en el trabajo.

Mi supervisor habla con optimismo sobre el futuro.

El comportamiento de mi supervisor es un ejemplo a seguir en la organización.

Se apoya a los trabajadores en el desarrollo de productos y/o servicios, la mejora de los procesos de innovación y la generación de nuevas ideas.

En nuestra empresa la innovación se acepta con facilidad por la gerencia.

En nuestra empresa nos ayudamos unos a otros para desarrollar nuestras actividades.

Las nuevas ideas son aceptadas rápidamente en nuestra empresa.

La empresa adopta las nuevas tecnologías en sus operaciones cotidianas.

La innovación es percibida como una oportunidad y es bien recibida en la empresa.

Solución de 26 variables a 4 componentes con eigenvalores superiores a 1 que explican el $70.1 \%$ de la variabilidad original. Método de extracción: Análisis de componentes principales. Método de rotación: Varimax con normalización Kaiser. Rotación convergió en 8 iteraciones.

Fuente: Desarrollado por los autores. 
Para el análisis factorial y lograr la separación de componentes, se utilizó una rotación varimax que es un método de rotación que minimiza el número de variables que tienen cargas altas en cada factor. El análisis estructural soporta bien, y en muchos casos asume, cierta correlación entre variables independientes latentes como un fenómeno común. De esta forma, al interpretar el contenido de los componentes con respecto a las cargas factoriales de cada uno de los atributos, se determinó que los componentes obtenidos son los siguientes: Liderazgo (X1), Aprendizaje organizacional (X2), Administración del conocimiento (X3), Innovación (Y). De estos cuatro componentes, tres corresponden a las dimensiones de aprendizaje organizacional, administración del conocimiento y liderazgo que fueron señalados en el planteamiento. Estas tres dimensiones se utilizan como variables latentes independientes en cada una de las tres hipótesis que fueron planteadas anteriormente. La dimensión número cuatro (innovación) correspondería a la variable latente dependiente en estas hipótesis. Los reactivos que se agrupan en estas cuatro dimensiones (variables latentes) se pueden ver en la Tabla 4.

Tabla 4

Reactivos (variables observables) por componentes (variables latentes) y fiabilidad de la escala de mediciones

\begin{tabular}{|c|c|c|}
\hline $\begin{array}{l}\text { Dimensión } \\
\text { (variable latente) }\end{array}$ & $\begin{array}{l}\text { Indicador } \\
\text { (variable observada) }\end{array}$ & $\operatorname{Alfa}(\alpha)$ \\
\hline \multirow[t]{8}{*}{ Liderazgo } & $\begin{array}{l}\text { Mi supervisor dedica tiempo a enseñarme y/o asesorarme en mis actividades } \\
\text { laborales. }\end{array}$ & .93 \\
\hline & $\begin{array}{l}\text { Hay suficiente confianza con mi supervisor para aceptar y retroalimentar sus } \\
\text { decisiones. }\end{array}$ & \\
\hline & Confío en las recomendaciones de mi supervisor para mejorar como empleado. & \\
\hline & La dirección de la empresa motiva al personal para alcanzar sus objetivos. & \\
\hline & $\begin{array}{l}\text { El gerente motiva a los empleados para mejorar las actividades desarrolladas en } \\
\text { la organización. }\end{array}$ & \\
\hline & $\begin{array}{l}\text { La organización cuenta con líderes que son capaces de motivar y guiar a sus } \\
\text { compañeros en el trabajo. }\end{array}$ & \\
\hline & Mi supervisor habla con optimismo sobre el futuro. & \\
\hline & El comportamiento de mi supervisor es un ejemplo a seguir en la organización. & \\
\hline \multirow{4}{*}{$\begin{array}{l}\text { Aprendizaje } \\
\text { Organizacional }\end{array}$} & Se conocen las fortalezas y debilidades del personal de la organización. & .82 \\
\hline & La empresa comparte los cambios a realizar en su operación. & \\
\hline & Se conocen los nuevos productos o servicios ofrecidos por nuestra empresa. & \\
\hline & $\begin{array}{l}\text { Todos los miembros de la organización comparten la misma meta hacia la cual se } \\
\text { sienten comprometidos. }\end{array}$ & \\
\hline \multirow[t]{4}{*}{$\begin{array}{l}\text { Administración del } \\
\text { Conocimiento }\end{array}$} & $\begin{array}{l}\text { Los empleados comparten conocimientos y experiencias en su área de trabajo } \\
\text { comunicándose entre ellos. }\end{array}$ & .91 \\
\hline & Evaluamos rápidamente las nuevas ideas que surgen en nuestra empresa. & \\
\hline & $\begin{array}{l}\text { Frecuentemente compartimos ideas con otras personas de intereses comunes } \\
\text { aunque estén en diferentes áreas. }\end{array}$ & \\
\hline & Se difunden nuevas ideas para mejorar la operación de la organización. & \\
\hline
\end{tabular}


Se realizan reuniones o juntas donde las personas comparten conocimientos y aprenden unos de otros.

Se comparte el conocimiento y experiencias entre los empleados de diferentes áreas.

Al compartir la información y el conocimiento frecuentemente generamos nuevas ideas para mejorar nuestro negocio.

La empresa cuenta con información actualizada de sus clientes atendidos.

$\begin{array}{lll}\text { Innovación } & \text { Se apoya a los trabajadores en el desarrollo de productos y/o servicios, la mejora } \quad .90\end{array}$ de los procesos de innovación y la generación de nuevas ideas.

En nuestra empresa la innovación se acepta con facilidad por la gerencia.

En nuestra empresa nos ayudamos unos a otros para desarrollar nuestras actividades.

Las nuevas ideas son aceptadas rápidamente en nuestra empresa.

La empresa adopta las nuevas tecnologías en sus operaciones cotidianas.

La innovación es percibida como una oportunidad y es bien recibida en la empresa.

Alfa $(\alpha)$ Coeficiente de fiabilidad interna Alfa de Cronbach

$\alpha$ de toda la escala $=.96$

Fuente: Desarrollado por los autores.

\section{Mediciones}

Para confirmar la confiabilidad en las mediciones se realizaron análisis de fiabilidad utilizando el alfa de Cronbach. Para estos análisis se obtuvo el índice de fiabilidad de los reactivos (atributos) que conforman la escala que considera las dimensiones obtenidas en el análisis factorial de la tabla 2 y que luego se utilizan como variables observadas en el análisis estructural. El alfa de Cronbach es un estadístico altamente confiable para determinar la consistencia interna de componentes de reactivos múltiples unidimensionales (Gliem y Gliem, 2003). En la tabla 3 se puede observar que en los reactivos asociados a las dimensiones (variables latentes) se obtuvo un coeficiente alfa que puede indicar altos niveles de consistencia interna en la medición según George y Mallery (2003), y Gliem y Gliem (2003).

Los reactivos utilizados para medir satisfacción son consistentes con aquellos utilizados en estudios previos de forma muy generalizada como puede verse en: Jiménez-Jiménez y SanzValle (2011), Wang y Lin (2013), García-Morales et al. (2012) y Tsai y Yang (2014) entre otros. Aunque se han propuesto diferentes modelos para medir la satisfacción, los reactivos utilizados en el presente estudio son consistentes con el enfoque propuesto por Noruzy et al. (2013), donde la innovación está precedida del aprendizaje organizacional, la administración del conocimiento y el liderazgo.

\section{Resultados del análisis descriptivo y modelo de regresión}

En la tabla 5 se muestran los resultados descriptivos del estudio. Dentro de las pruebas de estadística descriptiva se consideraron las medidas de tendencia central como la media, mediana, moda, desviación estándar, asimetría y curtosis. 
Asimismo, en la tabla se muestran los resultados de la prueba $t$ de diferencia de medias que sirve para verificar si las preguntas tienen la capacidad de discriminar las respuestas de los encuestados. Se compararon las medias del primer y el cuarto cuartil y se comprobó que las 26 preguntas obtuvieron un nivel de significancia menor a 0.05 .

Tabla 5

Resultados por reactivo. Media, desviación estándar, asimetría, curtosis y prueba t de diferencia de medias.

\begin{tabular}{|c|c|c|c|c|c|}
\hline Reactivo (variable observada) & Media & $\begin{array}{l}\text { Desviación } \\
\text { Estándar }\end{array}$ & Asimetría & Curtosis & $\mathrm{pdm}$ \\
\hline $\begin{array}{l}\text { Se apoya a los trabajadores en el desarrollo de } \\
\text { productos y/o servicios, la mejora de los procesos de } \\
\text { innovación y la generación de nuevas ideas. }\end{array}$ & 3.46 & 1.81 & .02 & -.70 & $*$ \\
\hline $\begin{array}{l}\text { En nuestra empresa la innovación se acepta con } \\
\text { facilidad por la gerencia. }\end{array}$ & 4.04 & 1.81 & -.20 & -.72 & $*$ \\
\hline $\begin{array}{l}\text { En nuestra empresa nos ayudamos unos a otros para } \\
\text { desarrollar nuestras actividades. }\end{array}$ & 4.38 & 1.90 & -.47 & -.93 & $*$ \\
\hline $\begin{array}{l}\text { Las nuevas ideas son aceptadas rápidamente en } \\
\text { nuestra empresa. }\end{array}$ & 3.90 & 1.74 & -.11 & -.51 & $*$ \\
\hline $\begin{array}{l}\text { La empresa adopta las nuevas tecnologías en sus } \\
\text { operaciones cotidianas. }\end{array}$ & 4.28 & 1.75 & -.48 & -.37 & $*$ \\
\hline $\begin{array}{l}\text { La innovación es percibida como una oportunidad y es } \\
\text { bien recibida en la empresa. }\end{array}$ & 4.19 & 1.78 & -.36 & -.42 & $*$ \\
\hline $\begin{array}{l}\text { Se conocen las fortalezas y debilidades del personal de } \\
\text { la organización. }\end{array}$ & 4.49 & 1.81 & -.53 & -.59 & $*$ \\
\hline $\begin{array}{l}\text { La empresa comparte los cambios a realizar en su } \\
\text { operación. }\end{array}$ & 4.48 & 1.90 & -.53 & -.56 & $*$ \\
\hline $\begin{array}{l}\text { Se conocen los nuevos productos o servicios ofrecidos } \\
\text { por nuestra empresa. }\end{array}$ & 4.18 & 1.98 & -.41 & -.81 & $*$ \\
\hline $\begin{array}{l}\text { Todos los miembros de la organización comparten la } \\
\text { misma meta hacia la cual se sienten comprometidos. }\end{array}$ & 4.53 & 1.77 & -.59 & -.33 & $*$ \\
\hline $\begin{array}{l}\text { Mi supervisor dedica tiempo a enseñarme y/o } \\
\text { asesorarme en mis actividades laborales. }\end{array}$ & 4.24 & 1.73 & -.24 & -.42 & $*$ \\
\hline $\begin{array}{l}\text { Hay suficiente confianza con mi supervisor para } \\
\text { aceptar y retroalimentar sus decisiones. }\end{array}$ & 4.99 & 1.63 & -.65 & -.25 & $*$ \\
\hline $\begin{array}{l}\text { Confío en las recomendaciones de mi supervisor para } \\
\text { mejorar como empleado. }\end{array}$ & 5.05 & 1.48 & -.78 & .29 & $*$ \\
\hline $\begin{array}{l}\text { La dirección de la empresa motiva al personal para } \\
\text { alcanzar sus objetivos. }\end{array}$ & 4.58 & 1.77 & -.66 & -.22 & $*$ \\
\hline $\begin{array}{l}\text { El gerente motiva a los empleados para mejorar las } \\
\text { actividades desarrolladas en la organización. }\end{array}$ & 4.30 & 1.81 & -.36 & -.68 & $*$ \\
\hline $\begin{array}{l}\text { La organización cuenta con líderes que son capaces de } \\
\text { motivar y guiar a sus compañeros en el trabajo. }\end{array}$ & 4.30 & 1.87 & -.32 & -.64 & $*$ \\
\hline Mi supervisor habla con optimismo sobre el futuro. & 4.74 & 1.87 & -.80 & -.14 & * \\
\hline $\begin{array}{l}\text { El comportamiento de mi supervisor es un ejemplo a } \\
\text { seguir en la organización. }\end{array}$ & 4.43 & 1.72 & -.57 & -.18 & $*$ \\
\hline
\end{tabular}


Los empleados comparten conocimientos y experiencias en su área de trabajo comunicándose entre ellos.

\begin{tabular}{lllll}
4.58 & 1.81 & -.47 & -.59 & $*$ \\
3.45 & 1.70 & .04 & -.25 & $*$ \\
3.81 & 1.72 & -.10 & -.59 & $*$ \\
3.86 & 1.73 & -.21 & -.31 & $*$ \\
3.33 & 1.98 & .31 & -.84 & $*$ \\
3.75 & 1.93 & -.10 & -.91 & $*$ \\
4.05 & 1.93 & -.09 & -.88 & $*$ \\
4.99 & 1.66 & -.93 & .75 & $*$ \\
\hline
\end{tabular}

Evaluamos rápidamente las nuevas ideas que surgen en nuestra empresa.

Frecuentemente compartimos ideas con otras personas de intereses comunes aunque estén en diferentes áreas.

Se difunden nuevas ideas para mejorar la operación de la organización.

Se realizan reuniones o juntas donde las personas comparten conocimientos y aprenden unos de otros.

Se comparte el conocimiento y experiencias entre los empleados de diferentes áreas.

Al compartir la información y el conocimiento frecuentemente generamos nuevas ideas para mejorar nuestro negocio.

La empresa cuenta con información actualizada de sus clientes atendidos.

pdm: Prueba t de diferencia de medias. Diferencias significativas * al .05.

Fuente: Desarrollado por los autores.

Para una primera aproximación a un análisis de causalidad se generó un modelo con análisis de regresión múltiple. Para este modelo la variable dependiente utilizada fue la medición de innovación (variables observadas de innovación). Como variables independientes se utilizaron las tres dimensiones de liderazgo, aprendizaje organizacional y administración del conocimiento obtenidas mediante el análisis factorial con rotación varimax que puede verse en las tablas 2 y 3 .

Tabla 6

Análisis de regresión lineal. Medición de innovación como variable dependiente. Coeficientes estandarizados.

\begin{tabular}{lll}
\hline & Y Innovación & $\mathrm{Z}$ \\
\hline $\mathrm{R}$ & .81 & \\
\hline $\mathrm{R}^{2}$ ajustada & .65 & \\
\hline $\mathrm{F}$ & $66.19 *$ & .07 \\
\hline $\mathrm{X}_{1}$ Liderazgo & $.39 *$ & $.11^{*}$ \\
\hline $\mathrm{X}_{2}$ Aprendizaje organizacional & $.31 *$ & .06 \\
\hline $\mathrm{X}_{3}$ Administración del conocimiento & $.22 *$ & \\
\hline
\end{tabular}

*Significativo al .05

Z: Prueba de normalidad Kolgomorov-Smirnov. H nula: La distribución es normal

Fuente: Desarrollado por los autores. 
Los resultados del análisis de regresión pueden verse en la tabla 6. Las tres variables independientes resultaron significativas. En la tabla 6 también se muestran indicadores de normalidad de las variables independientes utilizadas en el modelo de regresión lineal. Estos indicadores muestran que no todas las variables independientes cumplen con el criterio de normalidad.

\section{Resultados del análisis estructural}

Se hicieron dos modelos estructurales (modelos de ecuaciones estructurales) utilizando el software EQS V6.2. El modelo inicial de efectos directos contempló las hipótesis planteadas en las que se relacionan las tres variables latentes aprendizaje organizacional, administración del conocimiento y liderazgo con la variable latente de innovación (Ver figura 1). Se utilizaron los datos obtenidos de la aplicación de los cuestionarios como variables observadas. Estas variables observadas se relacionaron con cada una de sus respectivas tres variables latentes de acuerdo al análisis factorial que se muestra en la Tabla 1. Posteriormente se identificaron las variables latentes independientes con un mayor impacto significativo en la innovación y se generó el segundo modelo estructural que se le llamó modelo de efectos directos e indirectos.

Al realizar la prueba de normalidad de todas las variables observadas que se utilizaron se observó que no cumplen con el criterio de normalidad. Aun cuando el criterio de normalidad es un supuesto requerido para el análisis estructural existen evidencias que sugieren que cuando se tienen muestras grandes ( $\mathrm{n}=100$ o mayor), como en el presente caso, la no normalidad de las variables observadas tiende a no afectar la exactitud del modelo estructural (Jannoo et al. 2014).

En la Tabla 7 se puede observar que los modelos estructurales cumplen satisfactoriamente con los indicadores de ajuste requeridos para considerarlos como modelos aceptables. Los coeficientes de ajuste a la realidad (NFI, IFI y CFI) arriba de 0.90 se consideran aceptables, y por encima de 0.95 como buenos (Bentler y Bonett, 1980). Valores de 0.08 en el RMSEA se consideran aproximaciones de error razonables (aceptables) y valores de 0.05 o menores indican un buen ajuste con respecto a los grados de libertad, y se sugiere no aceptar valores mayores a 0.10 (Browne y Cudeck, 1993; MacCallum et al., 1996). En cuanto al indicador CMIN/DF (chi-cuadrada dividida entre los grados de libertad) valores menores a 5.0 pueden considerarse aceptables (Wheaton et al., 1977; Marsh y Hocevar, 1985). El RMSEA y el CMI/DF son medidas alternativas de ajuste para aceptar el modelo cuando la chi-cuadrada es demasiado grande como en este caso (Browne y Cudeck, 1993; MacCallum et al., 1996; Kenny, 2011).

Tabla 7

Modelos estructurales. Indicadores de ajuste.

\begin{tabular}{lcc}
\hline & $\begin{array}{c}\text { Modelo inicial } \\
\text { (Efectos directos) }\end{array}$ & $\begin{array}{c}\text { Modelo final } \\
\text { (Efectos directos e indirectos) }\end{array}$ \\
\hline Chi2 & 511.85 & 411.51 \\
\hline CMIN/DF & 1.72 & 1.39 \\
\hline BBNNFI & .83 & .91 \\
\hline IFI & .85 & .92 \\
\hline CFI & .85 & .92 \\
\hline RMSEA & .08 & .06 \\
\hline
\end{tabular}

Fuente: Desarrollado por los autores. 
En la Tabla 8 se puede ver los resultados de los pesos de regresión entre las variables latentes en los modelos estructurales. En el primer modelo se introdujeron las variables latentes independientes con efectos directos para determinar si existe un efecto sobre la variable latente innovación. Aquí las tres variables latentes independientes mostraron pesos de regresión estadísticamente significativos hacia la innovación. Liderazgo tuvo el valor del peso de regresión más alto.

Tabla 8

Análisis estructurales. Pesos de regresión estandarizados y coeficientes de determinación R2 para la variable dependiente (Innovación)

\begin{tabular}{lcc}
\hline Parámetros & $\begin{array}{c}\text { Modelo inicial } \\
\text { (Efectos directos) }\end{array}$ & $\begin{array}{c}\text { Modelo final } \\
\text { (Efectos directos e } \\
\text { indirectos) }\end{array}$ \\
\hline Aprendizaje ---> Innovación & .48 & .55 \\
\hline Conocimiento---> Innovación & .27 & -- \\
\hline Liderazgo---> Innovación & .52 & .36 \\
\hline Liderazgo--->Aprendizaje & -- & .41 \\
\hline Liderazgo--->Conocimiento & -- & .66 \\
\hline Conocimiento--->Aprendizaje & -- & .51 \\
\hline R2 para Innovación & .58 & .74 \\
\hline
\end{tabular}

* Significativo al .05. R2 = cuadrado de la correlación múltiple para la variable dependiente Fuente: Desarrollado por los autores.

En la Tabla 9 se puede ver un comparativo entre los coeficientes de determinación y los coeficientes de regresión de los análisis realizados.

Tabla 9

Coeficientes de determinación y coeficientes de regresión

\begin{tabular}{llcc}
\hline & & Innovación & Innovación (latente) \\
\hline Regresión lineal & R2 & .65 & \\
& X1 Liderazgo & $.39^{*}$ & \\
& X2 Aprendizaje organizacional & $.31^{*}$ & \\
\hline Análisis estructural & R2 Administración del conocimiento & $.22^{*}$ & .74 \\
\hline (modelo final) & X1 Aprendizaje ---> Y Innovación & $.55^{*}$ \\
\hline & X2 Conocimiento---> X1 Aprendizaje & $.51^{*}$ \\
\hline & X3 Liderazgo---> Y Innovación & $.36^{*}$ \\
\hline & X3 Liderazgo---> X1 Aprendizaje & $.41^{*}$ \\
\hline & X3 Liderazgo---> X2 Conocimiento & $.66^{*}$ \\
\hline
\end{tabular}

* Significativo al .05. Nota: Los coeficientes de determinación R2 no tienen asociado una prueba de significancia. Fuente: Desarrollado por los autores. 


\section{Conclusiones}

A diferencia de estudios anteriores sobre la innovación en empresas, el estudio realizado identifica factores que inciden en la innovación para que ésta se lleve a cabo (liderazgo, aprendizaje organizacional y administración del conocimiento). En el presente estudio se partió de una indagación exploratoria donde se realizó una detección detallada de atributos. De tal forma aquí se trabajó con 26 atributos específicos de aprendizaje organizacional, administración del conocimiento, liderazgo e innovación en empresas manufactureras y de servicios (Ver Tabla 2).

El haber detectado atributos muy específicos con base en el análisis del proceso de la innovación permite ofrecer una explicación más detallada de los generadores de la innovación del personal operativo en las empresas. El instrumento desarrollado permite aproximarse a la medición del efecto de los conceptos detectados como antecedentes en la innovación de las empresas.

Estudios anteriores se han abocado a la medición de la innovación en otros países desde el punto de vista del gerente o directivo de las empresas. En la revisión de la literatura no se encontraron estudios de innovación considerando la perspectiva del personal operativo en el contexto de las empresas mexicanas, al menos no en literatura de investigación académica. De esta forma, el presente estudio podría constituir una primera aproximación, a nivel de publicación académica, de los antecedentes de la innovación en las empresas mexicanas manufactureras y de servicios.

Tomando en cuenta que no existen estudios precedentes en innovación en el contexto mexicano, la expectativa es que el artículo apoye el desarrollo de nuevos trabajos que logren profundizar en el estudio de la innovación en el ámbito mexicano. La principal contribución del estudio radica en el análisis profundo que se realizó de los impulsores de la innovación; asimismo, las técnicas y metodologías utilizadas para el estudio empírico serán de utilidad para abordar estudios subsecuentes en el caso mexicano.

Es relevante abordar la percepción del personal operativo en México dado que el personal operativo es la base en la ejecución de la innovación y es el encargado de generar los productos y servicios que serán ofrecidos al consumidor.

En las tablas 10, 11, 12, 13 y Figuras 2 y 3 se muestran los resultados y validación de hipótesis del presente estudio.

Tabla 10

Validación de hipótesis del modelo de efectos directos.

\begin{tabular}{llllc}
\hline Modelo & Hipótesis & \multicolumn{1}{c}{ Camino } & Resultado & R-Cuadrada \\
\hline & H1 & Hacia Innovación de Liderazgo & Soportada & \\
\cline { 2 - 4 } Efectos Directos & H2 & $\begin{array}{l}\text { Hacia Innovación de Aprendizaje } \\
\text { Organizacional }\end{array}$ & Soportada \\
\cline { 2 - 4 } & H3 & $\begin{array}{l}\text { Hacia Innovación de Administración del } \\
\text { Conocimiento }\end{array}$ & Soportada \\
\hline
\end{tabular}

Fuente: Desarrollado por los autores. 
Tabla 11

Resultados del modelo de efectos directos en EQS.

\begin{tabular}{l}
\hline$l$ \\
Maximum Likelihood Solution \\
\hline Standardized Solution: \\
\hline $\mathrm{F} 1=\mathrm{F} 1=.489 * \mathrm{~F} 2+.522 * \mathrm{~F} 3+.276 * \mathrm{~F} 4+.642 \mathrm{D} 1$ \\
\hline $\begin{array}{l}(5.764 @ \quad(5.391 @ \quad(2.030 @ \\
\mathrm{INN}=.489 * \mathrm{AORG}+.522 * \mathrm{LID}+.276 * \mathrm{ACON}+.642 \mathrm{D} 1\end{array}$ \\
\hline
\end{tabular}

Coeficientes estandarizados $(\beta)$ : altos, @ = t-student $>1,96(\alpha=0,05)$, INN=Innovación, AORG=Aprendizaje organizacional, LID=Liderazgo, ACON=Administración del conocimiento.

Fuente: Desarrollado por los autores.

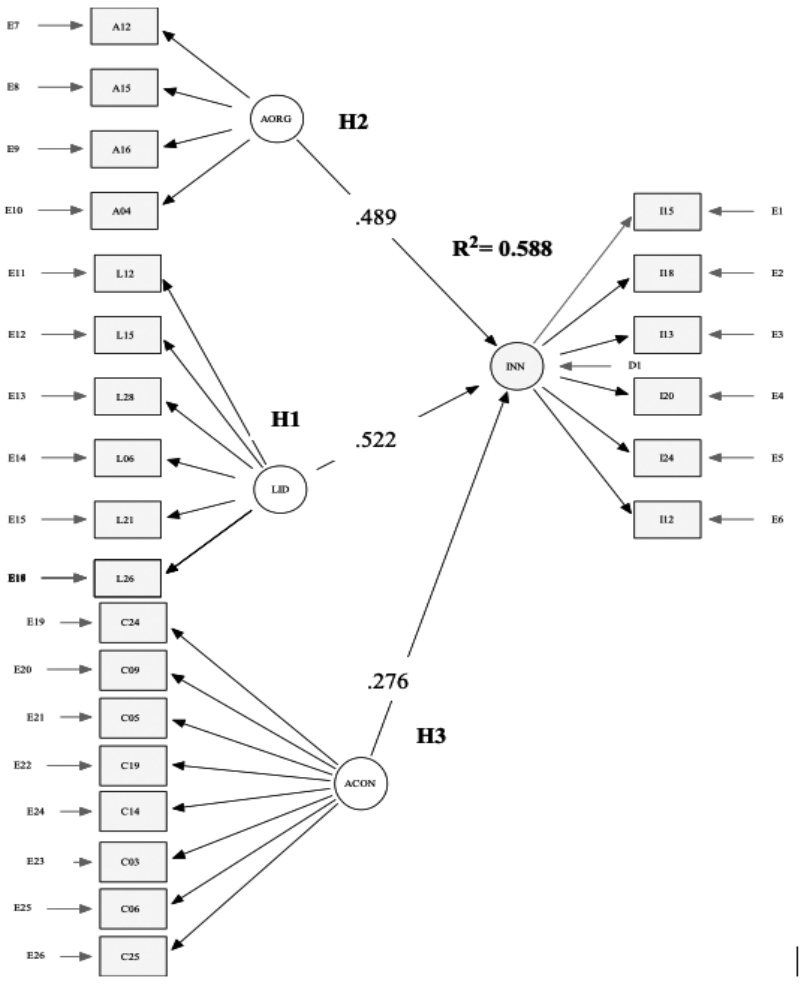

Figura 2. Resultados del modelo de efectos directos.

Fuente: Desarrollado por los autores. 
Tabla 12

Validación de hipótesis del modelo de efectos directos e indirectos.

\begin{tabular}{l|c|l|c|c|}
\hline Modelo & Hipótesis & \multicolumn{1}{|c|}{ Camino } & Resultado & R-Cuadrada \\
\hline & H4 & Hacia Innovación de Liderazgo & Soportada & \multirow{2}{*}{.74 } \\
\cline { 2 - 4 } Efectos Directos e Indirectos & H6 & $\begin{array}{l}\text { Hacia Aprendizaje Organizacional de } \\
\text { Liderazgo }\end{array}$ & $\begin{array}{l}\text { Sacia Administración del } \\
\text { Conocimiento de Liderazgo }\end{array}$ & Soportada \\
\cline { 2 - 4 } & H7 & $\begin{array}{l}\text { Hacia Aprendizaje Organizacional de } \\
\text { Liderazgo a través de Administración } \\
\text { del Conocimiento }\end{array}$ & Soportada \\
\cline { 2 - 4 } & H8 & $\begin{array}{l}\text { Hacia Innovación de Liderazgo } \\
\text { a través de Administración del } \\
\text { Conocimiento y Aprendizaje } \\
\text { Organizacional. }\end{array}$ & Soportada & \\
\hline
\end{tabular}

Fuente: Desarrollado por los autores.

Tabla 13

Resultados del modelo de efectos directos e indirectos en EQS.

\begin{tabular}{l|l}
\hline Maximum Likelihood Solution & \\
\hline Standardized Solution: & R-Squared \\
\hline $\mathrm{F} 1=\mathrm{F} 1=.555 * \mathrm{~F} 2+.362 * \mathrm{~F} 3+.508 \mathrm{D} 1$ & .742 \\
\hline $\begin{array}{c}(4.664 @(4.184 @ \\
\mathrm{F} 2=\mathrm{F} 2=.514 * \mathrm{~F} 4+.415 * \mathrm{~F} 3+.530 \mathrm{D} 2\end{array}$ \\
\hline $\begin{array}{c}(3.919 @(3.501 @ \\
\mathrm{F} 4=\mathrm{F} 4=.661 * \mathrm{~F} 3+.750 \mathrm{D} 4\end{array}$ \\
\hline $\mathrm{INN}=.555 * \mathrm{AORG}+.362 * \mathrm{LID}+.508 \mathrm{D} 1$ & .437 \\
\hline $\mathrm{AORG}=.514 * \mathrm{ACON}+.415 * \mathrm{LID}+.530 \mathrm{D} 2$ & .742 \\
\hline $\mathrm{ACON}=.661 * \mathrm{LID}+.750 \mathrm{D} 4$ & .719 \\
\hline
\end{tabular}

Coeficientes estandarizados $(\beta)$ : altos, @ = t-student $>1,96(\alpha=0,05)$, INN=Innovación, AORG=Aprendizaje organizacional, LID=Liderazgo, ACON=Administración del conocimiento.

Fuente: Desarrollado por los autores. 


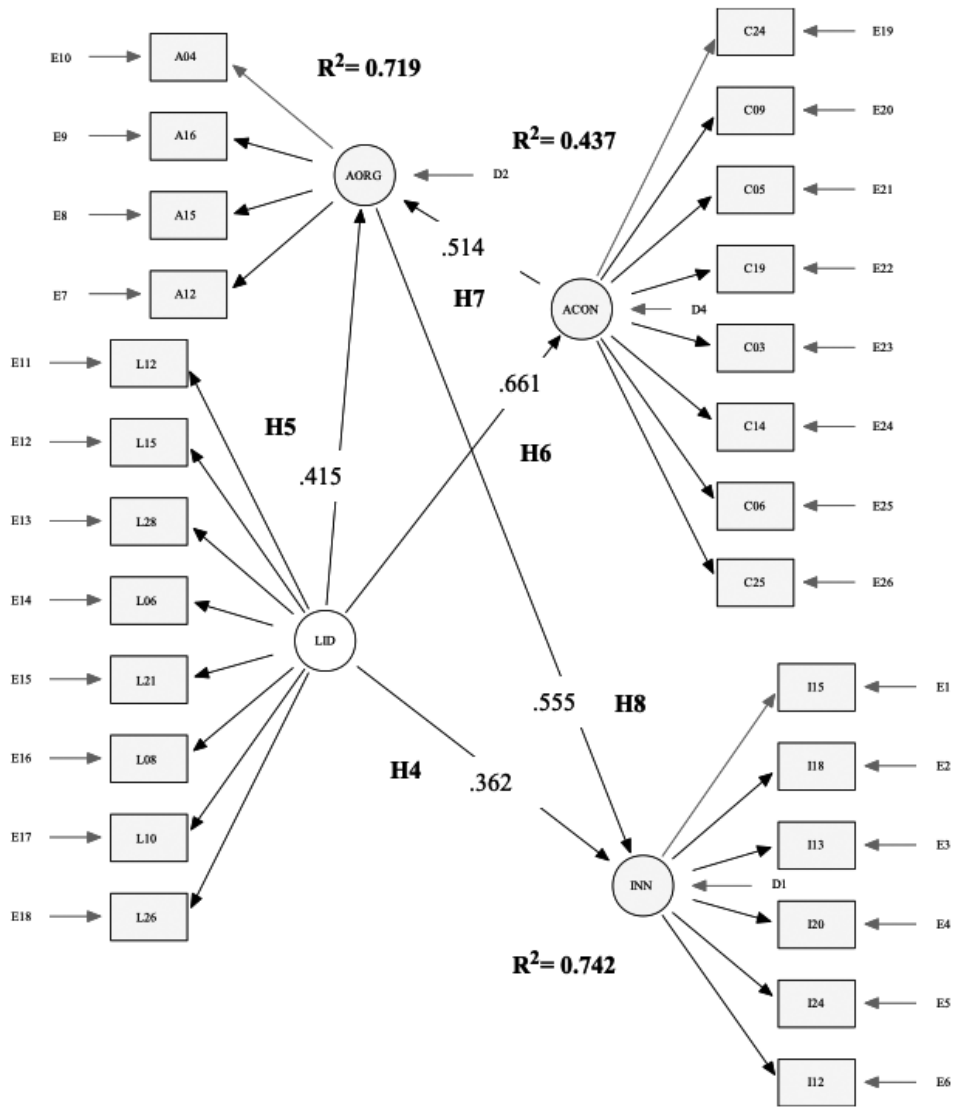

Figura 3. Resultados del modelo de efectos directos e indirectos (final).

Fuente: Desarrollado por los autores.

Con base en los resultados obtenidos, se puede argumentar que se tienen evidencias para soportar las hipótesis planteadas en el presente estudio tanto para el modelo de efectos directos como para el modelo de efectos directos e indirectos (final). Por lo que se puede decir que probablemente los factores, aprendizaje organizacional, administración del conocimiento y liderazgo, son los de importancia principal para generar una percepción de innovación por parte del personal operativo (ver Tabla 9). De estos, aparentemente el liderazgo juega un papel más preponderante que los otros. El factor liderazgo obtuvo los pesos estadísticos más altos en el modelo de regresión y en los modelos estructurales (ver Tabla 9).

Considerando que el análisis de ecuaciones estructurales en general es un método estadístico multivariado mucho más robusto que el análisis de regresión, se determinó para el presente estudio aceptar primordialmente los resultados del primero. De cualquier forma, siempre es interesante ver los resultados de dos métodos estadísticos distintos. 


\section{Limitaciones}

Con este estudio se ofrece un primer planteamiento sobre la relación directa entre dimensiones de aprendizaje organizacional, administración del conocimiento y liderazgo y la percepción de la innovación del personal operativo en las empresas mexicanas. No obstante, a pesar del esfuerzo realizado, el estudio presenta varias limitaciones. Una limitación es de tipo geográfica ya que se realizó con el personal operativo de empresas ubicadas en la zona metropolitana de la Ciudad de México. En investigaciones posteriores se podrían realizar estudios de innovación en empresas en otras regiones del país para evaluar los resultados por zona geográfica.

El trabajo desarrollado es un esfuerzo para determinar la incidencia que se tiene en la innovación considerando los tres constructos involucrados, por lo que es benéfico replicar este tipo de estudios en más empresas e instituciones a fin de fortalecer el instrumento desarrollado y obtener mayores evidencias empíricas que permitan generalizar el comportamiento del fenómeno.

Adicionalmente, existen algunos otros factores mencionados en la literatura sobre el tema que es posible tengan un efecto sobre la innovación en las empresas, pero que merecerían ser abordados en estudios adicionales que permitan profundizar en las relaciones observadas y sus efectos.

\section{Líneas de continuidad y profundización en la investigación de la innovación}

Investigaciones futuras pueden enfocarse en factores como el clima organizacional, la generación de conocimiento y la creatividad, entre otros. Estos factores pueden promover un ambiente favorable hacia la innovación y la formación de los llamados ecosistemas innovadores que impactan las organizaciones y los participantes que se encuentran cercanos a su operación.

Asimismo, los modelos de negocios que promueven la innovación y los nuevos emprendimientos de negocios pueden ser estudiados para determinar los factores que promueven el comportamiento innovador y derivar políticas que fomenten la innovación en las pequeñas y medianas empresas.

El estudio se centró en los precursores de la innovación; sin embargo, también se puede abordar la relación entre innovación y desempeño, lo cual es un aspecto que puede ser abordado en investigaciones posteriores para entender de forma más completa el fenómeno de la innovación y sus efectos en las organizaciones.

Los estudios más recientes en la literatura se relacionan con los conceptos abordados en la presente investigación. Para el caso del liderazgo, se identifica a la incertidumbre en la política presente en los países con elecciones nacionales como un factor que provoca una caída en las actividades de innovación tecnológica y al compromiso político por el contrario como un incentivador para la innovación (Bhattacharya et al., 2017). Al analizar las empresas manufactureras en una economía emergente (China), se encuentra que la estructura de propiedad con una participación mínima del estado resulta ser la estructura óptima para obtener los mejores resultados en el desarrollo de la innovación (Kevin Zheng et al., 2017). En el caso de las empresas familiares se observa un incremento en la tasa de conversión de los insumos a resultados de innovación en comparación con las empresas no familiares tomando en cuenta 
factores presentes en cada nación como la protección para los accionistas y el nivel de educación de la fuerza laboral (Duran et al., 2016). Se observa que el liderazgo transformador tiene un efecto positivo en el desempeño innovador del equipo de trabajo mediante los mecanismos integradores y centrados en el conocimiento como las normas cooperativas y la adquisición de conocimiento externo (Jiang y Chen, 2016). La motivación para la innovación se fundamenta en la creación de una cultura en la organización que tolere el fracaso temprano y recompense el desempeño a largo plazo mediante incentivos al personal (Manso, 2017).

En el caso del aprendizaje organizacional, al analizar los organismos intergubernamentales se observa un efecto positivo en la innovación nacional mediante la conectividad de las unidades de aprendizaje y el apalancamiento en el conocimiento externo (Jandhyala y Phene, 2015). Se resalta la importancia de las redes sociales para influir en la creatividad individual e innovación, identificando las fases para que una idea con un concepto novedoso pueda llegar a tener un resultado tangible (Perry-Smith y Mannucci, 2017). Se estudia el conocimiento tácito que puede extraerse de las subsidiarias de empresas multinacionales y los elementos mediadores como la eficacia de la tarea, la estructura orgánica y la confianza afectiva (Sheng et al., 2015).

En el caso de la administración del conocimiento se estudia el efecto de las tecnologías de información mediante el acceso a los sistemas de datos y las redes de conectividad para la absorción del conocimiento externo y la generación de estrategias para incrementar el desempeño innovador (Trantopoulos et al., 2017). Asimismo, se señala que las firmas enfrentan cuatro preocupaciones para asimilar plenamente la innovación digital: las capacidades existentes y requeridas, el enfoque en el producto o proceso, la colaboración interna o externa y la gobernanza es decir el control o la flexibilidad (Svahn et al., 2017). Adicionalmente, se observa que la administración y procesamiento de los flujos de la información del consumidor mediante las capacidades de absorción de información pueden incrementar los niveles de innovación en la firma (Saldanha et al., 2017).

Tomando en cuenta lo estudios recientes, podemos observar líneas adicionales de continuidad y profundización en la investigación de la innovación dirigidas hacia factores como las decisiones de política gubernamental, estructuras de propiedad de las empresas, funcionamiento de empresas familiares, liderazgo transformacional y generación de una cultura para la innovación. Asimismo, es importante el aprendizaje, la conectividad y el conocimiento externo, las redes sociales para la creatividad individual e innovación y la extracción del conocimiento tácito en las empresas. De igual manera es relevante el efecto de las tecnologías de información en la absorción del conocimiento externo, la asimilación de la innovación digital y las capacidades de absorción de información y sus efectos en la innovación de las empresas.

\section{Referencias}

Akman, G., \& Yilmaz, C. (2008). Innovative capability, innovation strategy and market orientation: an empirical analysis in Turkish software industry. International Journal of Innovation Management, 12(01), 69-111. doi:10.1142/ S1363919608001923.

Alexander, L., \& Van Knippenberg, D. (2014). Teams in pursuit of radical innovation: A Goal Orientation Perspective. Academy of Management Review, amr-2012. doi:10.5465/amr.2012.0044.

Anderson, N., Potocnik, K., \& Zhou, J. (2014). Innovation and Creativity in Organizations A State-of-the-Science Review, Prospective Commentary, and Guiding Framework. Journal of Management, 40(5), 1297-1333. doi: $10.1177 / 0149206314527128$. 
Bass, B.M. \& Avolio, B.J. (1999). Manual for the Multifactor Leadership Questionnaire (Form 5X). Palo Alto, CA: Mind Garden, Inc., Published by Mind Garden, Inc., www.mindgarden.com

Bentler, P.M. y Bonett, D.G. (1980). Significance tests and goodness of fit in the analysis of covariance structures. Psychological Bulletin, 88(1): 588-606. doi:10.1037/0033-2909.88.3.588

Bhattacharya, U., Hsu, P., Tian, X., \& Xu, Y. (2017). What Affects Innovation More: Policy or Policy Uncertainty? Journal Of Financial \& Quantitative Analysis, 52(5), 1869-1901. doi:10.1017/S0022109017000540.

Browne, M.W. y Cudeck, R. (1993). Alternative ways of assessing model fit. In Bollen, K.A. \& Long, J.S. [Eds.] Testing structural equation models. Newbury Park, CA: Sage: 136-162.

Capaldo, A., Lavie, D., \& Petruzzelli, A. M. (2014). Knowledge Maturity and the Scientific Value of Innovations: The Roles of Knowledge Distance and Adoption. Journal of Management, 0149206314535442. doi:10.1177/0149206314535442.

Cepeda-Carrion, G., Cegarra-Navarro, J. G., \& Jimenez-Jimenez, D. (2012). The Effect of Absorptive Capacity on Innovativeness: Context and Information Systems Capability as Catalysts. British Journal of Management, 23(1), 110-129. doi:10.1111/j.1467-8551.2010.00725.x.

Christensen, C. M. (1997). The Innovator's Dilemma: When New Technologies Cause Great Firms to Fail. Harvard Business Review Press.

Christensen, C. M., Raynor, M., \& McDonald, R. (2015). What is disruptive innovation?. Harvard Business Review, 93(12), 44-53.

Criscuolo, P., Salter, A., \& Ter Wal, A. L. (2013). Going underground: bootlegging and individual innovative performance. Organization Science. doi:10.1287/orsc.2013.0856.

Duran, P., Kammerlander, N., Van Essen, M., \& Zellweger, T. (2016). Doing more with less: innovation input and output in family firms. Academy Of Management Journal, 59(4), 1224-1264. doi:10.5465/amj.2014.0424.

Funk, R. J. (2014). Making the most of where you are: geography, networks, and innovation in organizations. Academy of Management Journal, 57(1), 193-222. doi:10.5465/amj.2012.0585.

García-Morales, V. J., Jiménez-Barrionuevo, M. M., Gutiérrez-Gutiérrez, L. (2012). Transformational leadership influence on organizational performance through organizational learning and innovation. Journal of Business Research, Volume 65, Issue 7, July, Pages 1040-1050. doi:10.1016/j.jbusres.2011.03.005.

García-Morales, V. J., Lloréns-Montes, F., \& Verdú-Jover, A. J. (2008). The Effects of Transformational Leadership on Organizational Performance through Knowledge and Innovation. British Journal of Management, 19(4), 299-319. doi:10.1111/j.1467-8551.2007.00547.x.

George, D., y Mallery, M. (2003). Using SPSS for Windows step by step: A simple guide and reference, Needham Heights, MA: Allyn \& Bacon.

Gliem, J. A. y Gliem, R. R. (2003). Calculating, interpreting, and reporting Cronbach's alpha reliability coefficient for Likert-type scales. Midwest Research-to-Practice Conference in Adult, Continuing, and Community Education, Columbus, OH: 82-88.

Graen, G., Novak, M. A., \& Sommerkamp, P. (1982). The effects of leader-member exchange and job design on productivity and satisfaction: Testing a dual attachment model. Organizational behavior and human performance, 30(1), 109-131.

Grigoriou, K., \& Rothaermel, F. T. (2014). Structural Microfoundations of Innovation: The Role of Relational Stars. Journal of Management, 40(2), 586-615. doi:10.1177/0149206313513612.

Hernández- Sampieri, R., Baptista, P., \& Fernández, C. (2014). Metodología de la Investigación. México: Mc Graw Hill.

Hu, B. (2014). Linking business models with technological innovation performance through organizational learning. European Management Journal, Volume 32, Issue 4, August 2014, Pages 587-595. doi:10.1016/j.emj.2013.10.009.

Jandhyala, S., \& Phene, A. (2015). The Role of Intergovernmental Organizations in Cross-border Knowledge Transfer and Innovation*. Administrative Science Quarterly, 60(4), 712-743. doi:10.1177/0001839215590153.

Jannoo, Z., Yap, B. W., Auchoybur, N., y Lazim, M. A. (2014). The Effect of Nonnormality on CB-SEM and PLS-SEM Path Estimates. International Journal of Mathematical, Computational, Natural and Physical Engineering, 8(2): 285-291. 
Jiang, Y. \& Chen, C. C. (2016). Integrating Knowledge Activities for Team Innovation: Effects of Transformational Leadership. Journal of Management, Published online before print March 2, 2016, doi:10.1177/0149206316628641.

Jiménez-Jiménez, D., \& Sanz-Valle, R. (2011). Innovation, organizational learning, and performance. Journal of Business Research, Volume 64, Issue 4, April, Pages 408-417. doi:10.1016/j.jbusres.2010.09.010.

Kenny, D.A. (2011). Measuring model fit. Disponible en: http://davidakenny.net/cm/fit.htm

Kerlinger, F. N. (1979). Behavioral research: A conceptual approach. New York: Holt, Rinehart, and Winston.

Kerlinger, F. N., \& Lee, H. B. (2002). Investigación del comportamiento. México City, México: McGraw-Hill.

Kevin Zheng, Z., Gerald Yong, G., \& Hongxin, Z. (2017). State Ownership and Firm Innovation in China: An Integrated View of Institutional and Efficiency Logics. Administrative Science Quarterly, 62(2), 375-404. doi: $10.1177 / 0001839216674457$.

MacCallum, R.C., Browne, M.W. y Sugawara, H.M. (1996). Power analysis and determination of sample size for covariance structure modeling. Psychological methods, 1(2): 130-149. doi:10.1037/1082-989X.1.2.130

Manso, G. (2017). Creating Incentives for Innovation. California Management Review, 60(1), 18-32. doi: $10.1177 / 0008125617725287$.

Marsh, H. W. y Hocevar, D. (1985). Application of confirmatory factor analysis to the study of self-concept: Firstand higher-order factor models and their invariance across groups. Psychological Bulletin, 97(1): 562-582. doi:10.1037/0033-2909.97.3.562

McKinley, W., Latham, S., \& Braun, M. (2014). Organizational decline and innovation: turnarounds and downward spirals. Academy of Management Review, 39(1), 88-110. doi:10.5465/amr.2011.0356.

Molina-Morales, F., García-Villaverde, P. M., \& Parra-Requena, G. (2014). Geographical and cognitive proximity effects on innovation performance in SMEs: A way through knowledge acquisition. International Entrepreneurship and Management Journal, 10(2), 231-251. doi:10.1007/s11365-011-0214-z.

Nissan, E., Galindo, M., Picazo, M. T., \& Méndez. (2012). Innovation, progress, entrepreneurship and cultural aspects. International Entrepreneurship and Management Journal, 8(4), 411-420. doi:10.1007/s11365-012-0229-0.

Noruzy, A., Dalfard, V., Azhdari, B., Nazari-Shirkouhi, S., \& Rezazadeh, A. (2013). Relations between transformational leadership, organizational learning, knowledge management, organizational innovation, and organizational performance: an empirical investigation of manufacturing firms. International Journal of Advanced Manufacturing Technology, 64(5-8), 1073-1085. doi:10.1007/s00170-012-4038-y.

Perry-Smith, J. E., \& Mannucci, P. V. (2017). From creativity to innovation: the social network drivers of the four phases of the idea journey. Academy Of Management Review, 42(1), 53-79. doi:10.5465/amr.2014.0462.

Rogan, M., \& Mors, M. L. (2014). A Network Perspective on Individual-Level Ambidexterity in Organizations. Organization Science. doi:10.1287/orsc.2014.0901.

Saldanha, T. V., Mithas, S., \& Krishnan, M. S. (2017). Leveraging customer involvement for fueling innovation: the role of relational and analytical information processing capabilities. MIS Quarterly, 41(1), 267-A11.

Scandura, T. A., \& Graen, G. B. (1984). Moderating effects of initial leader-member exchange status on the effects of a leadership intervention. Journal of applied psychology, 69(3), 428.

Schumpeter, J. A. (1934). The theory of economic development: An inquiry into profits, capital, credit, interest, and the business cycle. Cambridge, MA: Harvard University Press. Available at http://books.google.com.mx.

Schumpeter, J. A. (1942). Capitalism, socialism and democracy. New York: Harper \& Row. Available at http://books. google.com.mx.

Seidel, V. P., \& O’Mahony, S. (2014). Managing the Repertoire: Stories, Metaphors, Prototypes, and Concept Coherence in Product Innovation. Organization Science, 25(3), 691-712. doi:10.1287/orsc.2013.0879.

Sheng, M. L., Hartmann, N. N., Qimei, C., \& Chen, I. (2015). The Synergetic Effect of Multinational Corporation Management's Social Cognitive Capability on Tacit-Knowledge Management: Product Innovation Ability Insights from Asia. Journal Of International Marketing, 23(2), 94-110.

Svahn, F., Mathiassen, L., \& Lindgren, R. (2017). Embracing digital innovation in incumbent firms: how Volvo cars managed competing concerns. MIS Quarterly, 41(1), 239-254.

Trantopoulos, K., von Krogh, G., Wallin, M. W., \& Woerter, M. (2017). External knowledge and information technology: implications for process innovation performance. MIS Quarterly, 41(1), 287-A8. 
Tsai, K. H., \& Yang, S. Y. (2014). The contingent value of firm innovativeness for business performance under environmental turbulence. International Entrepreneurship and Management Journal, 10(2), 343-366. doi:10.1007/ s11365-012-0225-4.

Tuertscher, P., Garud, R., \& Kumaraswamy, A. (2014). Justification and Interlaced Knowledge at ATLAS, CERN. Organization Science. doi:10.1287/orsc.2013.0894.

Ugalde-Binda, N., Balbastre-Benavent, F., Canet-Giner, M. T., \& Escribá-Carda, N. (2014). The role of intellectual capital and entrepreneurial characteristics as innovation drivers. Innovar Journal Revista de Ciencias Administrativas y Sociales, 24(53), 41-60.

Verre, V., Petelski, N., \& Milesi, D. (2014). Cooperación y estrategia de apropiación en alta tecnología: el caso de una empresa biofarmacéutica argentina. Revista Innovar Journal Revista de Ciencias Administrativas y Sociales, 24(1Spe), 41-53.

Wallace, J. C., Butts, M. M., Johnson, P. D., Stevens, F. G., \& Smith, M. B. (2013). A Multilevel Model of Employee Innovation Understanding the Effects of Regulatory Focus, Thriving, and Employee Involvement Climate. Journal of Management. doi:10.1177/0149206313506462.

Wang, C., Rodan, S., Fruin, M., \& Xu, X. (2014). Knowledge networks, collaboration networks, and exploratory innovation. Academy Of Management Journal, 57(2), 454-514. doi:10.5465/amj.2011.0917.

Wang, Y., \& Lin, J. (2013). An empirical research on knowledge management orientation and organizational performance: The mediating role of organizational innovation. African Journal of Business Management, 7(8), 604-612. doi: 10.5897/AJBM11.2072.

Wheaton, B., Muthén, B., Alwin, D.F. y Summers, G.F. (1977). Assessing reliability and stability in panel models. In Heise, D.R. [Ed.] Sociological Methodology. San Francisco: Jossey-Bass: 84-136.

Wu, C. H., Parker, S. K., \& De Jong, J. P. (2014). Need for cognition as an antecedent of individual innovation behavior. Journal of Management, 40(6), 1511-1534. doi:10.1177/0149206311429862.

Yeung, A. L., Lai, K., \& Yee, R. Y. (2007). Organizational learning, innovativeness, and organizational performance: a qualitative investigation. International Journal of Production Research, 45(11), 2459-2477. doi:10.1080/00207540601020460. 